\title{
Decreasing the Value of Specified Cost Function by Adaptive Controller Based on Modified ACLF for a Class of Nonlinear Systems
}

\author{
Keizo Okano, ${ }^{1}$ Kojiro Hagino, ${ }^{2}$ and Hidetoshi Oya ${ }^{3}$ \\ ${ }^{1}$ Department of Systems Engineering, The University of Electro-Communications, 1-5-1 Chofugaoka, Chofu, Tokyo 182-8585, Japan \\ ${ }^{2}$ The University of Electro-Communications, 1-5-1 Chofugaoka, Chofu, Tokyo 182-8585, Japan \\ ${ }^{3}$ Institute of Technology and Science, The University of Tokushima, 2-1 Minamijosanjima, Tokushima 770-8506, Japan \\ Correspondence should be addressed to Keizo Okano; double_z_r@msn.com
}

Received 14 April 2014; Revised 30 August 2014; Accepted 19 October 2014; Published 24 November 2014

Academic Editor: Zoltan Szabo

Copyright (C) 2014 Keizo Okano et al. This is an open access article distributed under the Creative Commons Attribution License, which permits unrestricted use, distribution, and reproduction in any medium, provided the original work is properly cited.

\begin{abstract}
A new nonlinear adaptive control law for a class of uncertain nonlinear systems is proposed. The proposed control law is designed by a modified adaptive control Lyapunov function (ACLF) which satisfies a Hamilton-Jacobi-Bellman (HJB) equation. The modified ACLF is derived from transformation of an ACLF. The proposed control law is different from the inverse optimal one in decreasing the value of a cost function specified by a designer. In this paper, we show a transformation coefficient for an ACLF and a design method of a nonlinear adaptive controller. Finally, it is shown by a numerical simulation that the proposed control law decreases the value of a given cost function and achieves the desirable trajectory.
\end{abstract}

\section{Introduction}

Design of control laws considering stability and optimality is the central issue in control theory [1]. For stability, Lyapunov theory is a strong tool to design controllers and to assure the stability of systems. For optimality, a value function which is the solution to a Hamilton-Jacobi-Bellman (HJB) equation is derived from dynamic programming. If a value function and an optimal control law can be found, then the closed system possesses robustness such as gain margin, phase margin, and low sensitivity against parameter variations $[2,3]$.

However, a general approach to find the value function has not been shown and it is not easy to design the optimal control. Due to the difficulty, the inverse optimal control problem which minimizes a meaningful cost function was proposed by Freeman and Kokotovic. If the inverse optimal problem is solved, namely, a control Lyapunov function (CLF) is found, it is possible to design a control law with the good characteristics mentioned above by applying a CLF to the Pointwise Min-Norm (PMN) control law [4]. But the minimized cost function and the trajectory may not be desirable. In order to improve this problem, a locally approximate approach around the origin by numerical calculation and transformation of a CLF was proposed. The approach gives characteristics of local optimality without loss of characteristics of the global inverse optimality $[5,6]$, and it is based on the fact that the PMN control law can minimize the desired cost function if a CLF has the same level sets as the value function [7].

The inverse optimal control law was applied to robust control and adaptive control $[8,9]$. Moreover, a control law in which a Sontag type control law and a PMN control law were generalized has been proposed [10-12]. Also, many approaches to approximate the value function have been proposed. They are based on numerical calculation and focus on improvement of calculation speed and accuracy of approximation $[5,13]$.

Recently, a new approach has been provided by [14]. The new approach in [14] is different from the inverse optimal and approximate approaches in directly considering a cost function specified by a designer and provides a modified ACLF by introducing a transformation coefficient. This paper expands [14] and proves a modified ACLF and a control 
law designed by a modified ACLF minimize the value of a cost function specified by a designer in the set of control laws based on an ACLF. Also, the condition under which a controller and a transformation coefficient are continuous is provided and the problem of overparameterization which is to increase the number of parameter estimation according to the order of a system is solved.

In this paper, the design of a modified ACLF and a nonlinear adaptive control law is shown in Sections 3 and 4 and effectiveness of the proposed controller is shown by a numerical simulation in Section 5.

The following notations are used in this paper. $\mathbb{R}$ denotes the sets of real value. $\mathbb{R}^{+}$denotes the sets of positive real value. $\mathscr{V}_{x}(x)$ denotes the derivative of $\mathscr{V}(x)$ with respect to $x$. For a matrix $\mathscr{A}, \mathscr{A}>0(\geq 0)$ denotes that a matrix $\mathscr{A}$ is positive definite (semipositive definite) matrix. $\mathscr{A}^{T}$ denotes transpose of matrix $\mathscr{A}$.

\section{Preliminaries}

We deal with a nonlinear strict-feedback system with unknown parameters described by

$$
\begin{aligned}
\dot{x}_{i}(t)= & f_{i}\left(x_{1}, \ldots, x_{i}\right)+F_{i}^{T}\left(x_{1}, \ldots, x_{i}\right) \theta \\
& +g_{i}\left(x_{1}, \ldots, x_{i}\right) x_{i+1}(t), \\
\dot{x}_{n}(t)= & f_{n}\left(x_{1}, \ldots, x_{n}\right)+F_{n}^{T}\left(x_{1}, \ldots, x_{n}\right) \theta \\
& +g_{n}\left(x_{1}, \ldots, x_{n}\right) u(t),
\end{aligned}
$$

where $i=1, \ldots, n-1, x_{i}(t) \in \mathbb{R}, u(t) \in \mathbb{R}$, and $\theta \in \mathbb{R}^{p}$ denote the state, the control input, and a vector of unknown constant parameters, respectively. It is assumed in this paper that all information of the state $x(t)$ can be used. $f_{i}\left(x_{1}(t), \ldots, x_{i}(t)\right)$ : $\mathbb{R}^{i} \rightarrow \mathbb{R}$ and $F_{i}\left(x_{1}(t), \ldots, x_{i}(t)\right): \mathbb{R}^{i} \rightarrow \mathbb{R}^{p}$ satisfy $f_{i}(0)=0$ and $F_{i}(0)=0$, respectively. $g_{i}\left(x_{1}(t), \ldots, x_{i}(t)\right): \mathbb{R}^{i} \rightarrow \mathbb{R}$ is not equal to 0 for all $x$. In compact notation, the system (1) is described as

$$
\dot{x}(t)=f(x)+F^{T}(x) \theta+g(x) u(t),
$$

where $f(x)=\left[f_{1}\left(x_{1}\right)+g_{1}\left(x_{1}\right) x_{2}(t), \ldots, f_{i}\left(x_{1}, \ldots, x_{i}\right)+\right.$ $\left.g_{i}\left(x_{1}, \ldots, x_{i}\right) x_{i+1}(t), \ldots, f_{n}\left(x_{1}, \ldots, x_{n}\right)\right]^{T}, F(x)=\left[F_{1}\left(x_{1}\right)\right.$, $\left.\ldots, F_{n}\left(x_{1}, \ldots, x_{n}\right)\right]^{T}$, and $g(x)=\left[0, \ldots, 0, g_{n}\left(x_{1}, \ldots, x_{n}\right)\right]^{T}$ are smooth and $f(0)=0, F(0)=0$, so that $x=0$ is an equilibrium of the uncontrolled plant. In order to estimate the unknown parameter $\theta \in \mathbb{R}^{p}$, the following adaptive law is introduced:

$$
\dot{\hat{\theta}}(t)=\Gamma \tau(x, \widehat{\theta}),
$$

where $\widehat{\theta} \in \mathbb{R}^{p}$ is the estimation of the parameter $\theta, \Gamma=\Gamma^{T}>$ $0 \in \mathbb{R}^{p \times p}$ is a constant parameter specified by a designer, and $\tau(x, \widehat{\theta}): \mathbb{R}^{n+p} \rightarrow \mathbb{R}^{p}$ is the tuning function. The construction of the tuning function is explained in Section 4.
The cost function is defined as

$$
\begin{aligned}
\mathscr{J} & =\int_{0}^{\infty} \mathscr{L}(x, u) d t \\
& =\int_{0}^{\infty}\left(q(x)+r(x) u^{2}(t)\right) d t,
\end{aligned}
$$

where $q(x): \mathbb{R}^{n} \rightarrow \mathbb{R}$ and $r(x): \mathbb{R}^{n} \rightarrow \mathbb{R}$ are weighting functions and satisfy $q(x)>0$ for all $x \neq 0, q(0)=0$, and $r(x)>0$ for all $x$.

It is well known that the optimal control problem which minimizes the cost function (4) is reduced to the problem to find the value function satisfying the following HJB equation [10]:

$$
\begin{aligned}
\overline{\mathscr{H}}(x, \widehat{\theta})= & \mathscr{L}(x, \bar{u}(t))+\dot{\overline{\mathscr{V}}}(x, \widehat{\theta}) \\
= & q(x)+\overline{\mathscr{V}}_{x}^{T}(x, \widehat{\theta})\left(f(x)+F^{T}(x) \theta\right) \\
& +\overline{\mathscr{V}}_{\widehat{\theta}}^{T}(x, \widehat{\theta}) \Gamma \tau(x, \widehat{\theta}) \\
& -\frac{1}{4 r(x)} \overline{\mathscr{V}}_{x}^{T}(x, \widehat{\theta}) g(x) g^{T}(x) \overline{\mathscr{V}}_{x}(x, \widehat{\theta}) \\
= & 0,
\end{aligned}
$$

where the optimal control law $\bar{u}(t)$ is given by

$$
\bar{u}(t)=-\frac{1}{2 r(x)} g^{T}(x) \overline{\mathscr{V}}_{x}(x, \widehat{\theta}) .
$$

However, it is generally difficult to solve the HJB equation and to find the value function. In this paper, assuming that there exists an ACLF for the system (1), a certain transformation coefficient is introduced and a nonlinear adaptive control law is constructed by a modified ACLF based on an original ACLF.

\section{Main Results}

In this section, we show the controller based on a modified ACLF by a certain transformation coefficient.

First, an ACLF with unknown parameters and an adaptive law is defined as the following definition.

Definition 1. $\mathscr{V}(x, \widehat{\theta}): \mathbb{R}^{n+p} \rightarrow \mathbb{R}^{+}$is called an ACLF for the system (2) and (3) if it is a positive definite function which is continuous and radially unbounded and satisfies the following inequality:

$$
\begin{gathered}
\inf _{u \in \mathbb{R}}\left[\mathscr{V}_{x}^{T}(x, \widehat{\theta})\left(f(x)+F^{T}(x) \theta\right)+\mathscr{V}_{\widehat{\theta}}^{T}(x, \widehat{\theta}) \Gamma \tau(x, \widehat{\theta})\right. \\
\left.+\mathscr{V}_{x}^{T}(x, \widehat{\theta}) g(x) u(t)\right]<0 \quad \forall x \neq 0 .
\end{gathered}
$$

The following theorem gives the transformation coefficient for the modified ACLF and the control law based on an ACLF.

Theorem 2. It is assumed that there exists an ACLF $\mathscr{V}(x, \hat{\theta})$ for the system (2) and (3). Let $\widetilde{\mathscr{V}}(x, \widehat{\theta})$ be

$$
\widetilde{\mathscr{V}}(x, \widehat{\theta})=\int_{0}^{t}\left(k(x, \widehat{\theta}) \frac{d}{d t} \mathscr{V}(x, \widehat{\theta})\right) d t+C,
$$


where $k(x, \hat{\theta})$ is defined by

$$
\begin{gathered}
k(x, \hat{\theta})=2 r(x) \frac{\sigma_{1}(x, \widehat{\theta})+\sigma_{2}(x, \hat{\theta})}{\mathscr{V}_{x}^{T}(x, \widehat{\theta}) g(x) g^{T}(x) \mathscr{V}_{x}(x, \widehat{\theta})} \\
\left(\mathscr{V}_{x}^{T}(x, \hat{\theta}) g(x) \neq 0\right), \\
k(x, \hat{\theta})=-\frac{q(x)}{\sigma_{1}(x, \widehat{\theta})} \quad\left(\mathscr{V}_{x}^{T}(x, \hat{\theta}) g(x)=0\right), \\
k(x, \hat{\theta})=0 \quad(x=0)
\end{gathered}
$$

and $\sigma_{1}(x, \widehat{\theta})$ and $\sigma_{2}(x, \widehat{\theta})$ in $(9 a)-(9 c)$ are scalar functions defined by

$$
\begin{aligned}
\sigma_{1} & (x, \hat{\theta}) \\
& =\mathscr{V}_{x}^{T}(x, \widehat{\theta})\left(f(x)+F^{T}(x) \theta\right)+\mathscr{V}_{\widehat{\theta}}^{T}(x, \widehat{\theta}) \Gamma \tau(x, \widehat{\theta}),
\end{aligned}
$$

$$
\begin{aligned}
& \sigma_{2}(x, \hat{\theta}) \\
& =\sqrt{\left(\sigma_{1}(x, \widehat{\theta})\right)^{2}+\frac{q(x)}{r(x)} \mathscr{V}_{x}^{T}(x, \widehat{\theta}) g(x) g^{T}(x) \mathscr{V}_{x}(x, \widehat{\theta})},
\end{aligned}
$$

respectively. $C$ denotes a constant and is sufficiently large so that $\widetilde{\mathscr{V}}(x, \widehat{\theta})$ is a positive definite function. Then, $k(x, \widehat{\theta})>0$ for all $x \neq 0$ and $\widetilde{\mathscr{V}}(x, \widehat{\theta})$ satisfies HJB equation (5) with the following control input:

$$
u(t)=-\frac{k(x, \widehat{\theta})}{2 r(x)} g^{T}(x) \mathscr{V}_{x}(x, \widehat{\theta})
$$

and the closed system with the control input (12) is asymptotically stable to the origin.

Proof. First, we show that $k(x, \widehat{\theta})$ is given by $(9 a)-(9 c)$ so that $\widetilde{\mathscr{V}}(x, \widehat{\theta})$ satisfies HJB equation and $k(x, \widehat{\theta})>0$ for all $x \neq 0$. In HJB equation (5), when $\dot{\widetilde{\mathscr{V}}}(x, \widehat{\theta})$ is applied instead of the value function $\dot{\overline{\mathscr{V}}}(x, \widehat{\theta})$, the quadratic equation in terms of $k(x, \widehat{\theta})$ is derived by

$$
\begin{aligned}
\mathscr{H}(x, \widehat{\theta})= & \mathscr{L}(x, u)+\dot{\widetilde{\mathscr{V}}}(x, \widehat{\theta}) \\
= & q(x)+r(x) u^{2}(t) \\
& +k(x, \widehat{\theta})\left(\sigma_{1}(x, \widehat{\theta})+\mathscr{V}_{x}^{T}(x, \widehat{\theta}) g(x) u(t)\right) \\
= & q(x)+k(x, \widehat{\theta}) \sigma_{1}(x, \hat{\theta}) \\
& -\frac{k^{2}(x, \widehat{\theta})}{4 r(x)} \mathscr{V}_{x}^{T}(x, \widehat{\theta}) g(x) g^{T}(x) \mathscr{V}_{x}(x, \widehat{\theta}) \\
= & 0,
\end{aligned}
$$

where input $u(t)$ is given by (12) from $\mathscr{H}_{u}(x, \widehat{\theta})=0$. Equation (13) can easily be solved with respect to $k(x, \widehat{\theta})$; namely, $k(x, \widehat{\theta})=\left(-b \pm \sqrt{b^{2}-4 a c}\right) / 2 a$ in the case of $a \neq$ 0 and $k(x, \widehat{\theta})=-c / b$ in the case of $a=0$ where $a=$ $\mathscr{V}_{x}^{T}(x, \widehat{\theta}) g(x) g(x)^{T} \mathscr{V}_{x}(x, \widehat{\theta}), b=\sigma_{1}(x, \widehat{\theta})$, and $c=q(x)$ and the solution becomes

$$
\begin{gathered}
k(x, \hat{\theta})=2 r(x) \frac{\sigma_{1}(x, \hat{\theta}) \pm \sigma_{2}(x, \hat{\theta})}{\mathscr{V}_{x}^{T}(x, \widehat{\theta}) g(x) g^{T}(x) \mathscr{V}_{x}(x, \widehat{\theta})} \\
\left(\mathscr{V}_{x}^{T}(x, \widehat{\theta}) g(x) \neq 0\right), \\
k(x, \hat{\theta})=-\frac{q(x)}{\sigma_{1}(x, \widehat{\theta})} \quad\left(\mathscr{V}_{x}^{T}(x, \hat{\theta}) g(x)=0\right) .
\end{gathered}
$$

Though there are two solutions in (14a) when $\mathscr{V}_{x}^{T}(x, \widehat{\theta}) g(x) \neq$ 0 , we select one of them as (9a). Then, $k(x, \widehat{\theta})>0$ for all $x \neq 0$ because, in the case that $\mathscr{V}_{x}^{T}(x, \widehat{\theta}) g(x) \neq 0, \sigma_{1}(x, \widehat{\theta}) \leq$ $\sqrt{\left(\sigma_{1}(x, \hat{\theta})\right)^{2}}<\sigma_{2}(x, \hat{\theta})$ for all $x \neq 0$ and, in the case that $\mathscr{V}_{x}^{T}(x, \widehat{\theta}) g(x)=0$, since $\mathscr{V}(x, \widehat{\theta})$ is an ACLF which satisfies (7), $\sigma_{1}(x, \widehat{\theta})<0$ for all $x \neq 0$. Therefore, we obtain $k(x, \widehat{\theta})>0$ for all $x \neq 0$.

Next, we will show that the system is asymptotically stable by the control law (9a)-(9c) and (12). In the case that $\mathscr{V}_{x}^{T}(x, \widehat{\theta}) g(x) \neq 0$, the time derivative of $\mathscr{V}(x, \widehat{\theta})$ is given by (16a). Since $\sigma_{2}(x, \widehat{\theta})>0$, we obtain $\dot{\mathscr{V}}(x, \widehat{\theta})<0$. In the case that $\mathscr{V}_{x}^{T}(x, \widehat{\theta}) g(x)=0$, the time derivative of $\mathscr{V}(x, \widehat{\theta})$ is given by (16b). Consider

$$
\begin{aligned}
\dot{\mathscr{V}}(x, \widehat{\theta})= & \mathscr{V}_{x}^{T}(x, \hat{\theta})\left(f(x)+F^{T}(x) \theta\right) \\
& +\mathscr{V}_{\hat{\theta}}^{T}(x, \widehat{\theta}) \Gamma \tau(x, \hat{\theta})+\mathscr{V}_{x}^{T}(x, \hat{\theta}) g(x) u(t),
\end{aligned}
$$

$$
\begin{gathered}
\dot{\mathscr{V}}(x, \hat{\theta})=-\sigma_{2}(x, \hat{\theta}) \quad\left(\mathscr{V}_{x}^{T}(x, \widehat{\theta}) g(x) \neq 0\right), \\
\dot{\mathscr{V}}(x, \hat{\theta})=\sigma_{1}(x, \hat{\theta}) \quad\left(\mathscr{V}_{x}^{T}(x, \widehat{\theta}) g(x)=0\right) .
\end{gathered}
$$

$\sigma_{1}(x, \widehat{\theta})<0$ because $\mathscr{V}(x, \widehat{\theta})$ is an ACLF which satisfies (7). Therefore we also obtain $\dot{\mathscr{V}}(x, \widehat{\theta})<0$ in the case of $(16 \mathrm{~b})$ and $\dot{\mathscr{V}}(x, \widehat{\theta})<0$ is satisfied for all $x \neq 0$. As a result, $\mathscr{V}(x, \widehat{\theta})$ is the ACLF for the system (2) and (3) with the input (9a)(9c) and (12). We show that $\widetilde{\mathscr{V}}(x, \widehat{\theta})$ is also an ACLF. It is trivial that the time derivative of $\widetilde{\mathscr{V}}(x, \widehat{\theta})$ is $k(x, \widehat{\theta}) \dot{\mathscr{V}}(x, \widehat{\theta})<0$ for all $x \neq 0$ because $k(x, \widehat{\theta})>0$ and $\dot{\mathscr{V}}(x, \widehat{\theta})<0$ for all $x \neq 0$. Since $\widetilde{\mathscr{V}}(x(t), \widehat{\theta}(t))$ changes its value with $t$ similarly as $\mathscr{V}(x(t), \hat{\theta}(t))$ and $\dot{\widetilde{\mathscr{V}}}(x(\infty), \hat{\theta}(\infty))=0$ and there always exists $C$ to make $\widetilde{\mathscr{V}}(x(\infty), \hat{\theta}(\infty))=0$, we can have $\widetilde{\mathscr{V}}(x, \widehat{\theta})$ be positive definite for all $x \neq 0$. Therefore, $\widetilde{\mathscr{V}}(x, \widehat{\theta})$ is an ACLF for the system. Therefore, the solution of the system (2) and (3) converges to the maximum invariant sets $\mathcal{S}$ included in the set $\mathcal{U}=\{x \mid \dot{\widetilde{\mathscr{V}}}(x, \widehat{\theta})=0\}$ by LaSalle's invariance principle [15]. Since the invariant sets $\mathcal{S}$ consist of the origin only, the closed system is asymptotically stable to the origin. 
In Theorem 2, the ACLF $\mathscr{V}(x, \hat{\theta})$ is modified by the transformation coefficient $k(x, \widehat{\theta})$ and the modified $\widetilde{\mathscr{V}}(x, \widehat{\theta})$ is an ACLF and satisfies HJB equation.

Theorems about the cost function (4), the transformation coefficient $k(x, \widehat{\theta})$, and the control input (12) are given as follows.

Corollary 3. The value of cost function is given by the following equation if the transformation coefficient (9a)-(9c) and control law (12) are applied:

$$
\mathscr{J}=-\int_{0}^{\infty} \dot{\overline{\mathscr{V}}}(x, \hat{\theta}) d t .
$$

Proof. Equation (18) is given from (13):

$$
\mathscr{L}(x, u)=-\dot{\widetilde{\mathscr{V}}}(x, \widehat{\theta}) .
$$

By integrating of (18) from 0 to $\infty$, we obtain

$$
\int_{0}^{\infty} \mathscr{L}(x, u) d t=-\int_{0}^{\infty} \dot{\dot{\mathscr{V}}}(x, \hat{\theta}) d t .
$$

Theorem 4. The control law (12) with (9a)-(9c) minimizes the cost function (4) in the set of control laws based on the ACLF $\mathscr{V}(x, \widehat{\theta})$.

Proof. It is assumed that the control (20) stabilizes the system:

$$
u(t)=v(t)-\frac{k(x, \widehat{\theta})}{2 r(x)} g^{T}(x) \mathscr{V}_{x}(x, \hat{\theta}) .
$$

By substituting (20) for the cost function (4) and applying (13), we obtain

$$
\begin{aligned}
& \mathscr{J}=\int_{0}^{\infty}\{q(x)+r(x) \\
& \left.\times\left(v(t)-\frac{k(x, \widehat{\theta})}{2 r(x)} g^{T}(x) \mathscr{V}_{x}(x, \widehat{\theta})\right)^{2}\right\} d t \\
& =-\int_{0}^{\infty} k(x, \widehat{\theta}) \mathscr{V}_{x}^{T}(x, \widehat{\theta}) \\
& \times\left\{f(x)+F^{T}(x) \theta\right. \\
& \left.+g(x)\left(v-\frac{k(x, \widehat{\theta})}{2 r(x)} g^{T}(x) \mathscr{V}_{x}(x, \widehat{\theta})\right)\right\} d t \\
& -\int_{0}^{\infty} k(x, \widehat{\theta}) \mathscr{V}_{\widehat{\theta}}^{T}(x, \widehat{\theta}) \Gamma \tau(x, \widehat{\theta}) d t \\
& +\int_{0}^{\infty} r(x) v(t)^{2} d t \\
& =-\int_{0}^{\infty} k(x, \widehat{\theta}) \dot{\mathscr{V}}(x, \widehat{\theta}) d t+\int_{0}^{\infty} r(x) v(t)^{2} d t .
\end{aligned}
$$

Since the first term in (21) is positive, (21) is minimized when $v(t)=0$. Therefore, the cost function (4) is minimized by the control law (12) using the $\operatorname{ACLF} \mathscr{V}(x, \widehat{\theta})$.

Theorem 5. If $\sigma_{1}(x, \widehat{\theta}), \mathscr{V}_{x}^{T}(x, \hat{\theta}) g(x), q(x)$, and $r(x)$ are continuous and differentiable for all $x \neq 0$, then transformation coefficient (9a)-(9c) is continuous and differentiable for all $x \neq$ 0 .

Proof. $\mathscr{H}(x, \widehat{\theta})$ of (13) can be regarded as the function of $x(t)$ and $k(x, \widehat{\theta})$, namely, $\mathscr{H}(x, k)$. The derivative of the function $\mathscr{H}(x, k)$ with respect to $k(x, \widehat{\theta})$ is given by

$$
\begin{gathered}
\mathscr{H}_{k}(x, k(x, \hat{\theta}))=-\sigma_{2}(x, \hat{\theta}) \quad\left(\mathscr{V}_{x}^{T}(x, \hat{\theta}) g(x) \neq 0\right), \\
\mathscr{H}_{k}(x, k(x, \hat{\theta}))=\sigma_{1}(x, \widehat{\theta}) \quad\left(\mathscr{V}_{x}^{T}(x, \hat{\theta}) g(x)=0\right),
\end{gathered}
$$

where we used (9a)-(9c). In (22a), $\sigma_{2}(x, \widehat{\theta})>0$ for all $x \neq 0$. In $(22 \mathrm{~b}), \sigma_{1}(x, \widehat{\theta})<0$ for all $x \neq 0$ since $\mathscr{V}(x, \widehat{\theta})$ is an ACLF. Therefore, $\mathscr{H}_{k}(x, k)<0$ for all $x \neq 0$. From implicit function theorem, $k(x, \widehat{\theta})$ is continuous and differentiable for all $x \neq 0$ if $\sigma_{1}(x, \widehat{\theta}), \mathscr{V}_{x}^{T}(x, \widehat{\theta}) g(x), q(x)$, and $r(x)$ are continuous and differentiable for all $x \neq 0$.

Remark 6. It is assumed that the weighting function $q(x)>0$ for all $x \neq 0$ in this paper. But even if $\sigma_{1}(x, \widehat{\theta}) \neq 0$ when $q(x)=0$ for all $x \neq 0$, the proposed approach can also be applied (refer to Section 5).

Remark 7. The control law includes the unknown parameters in (10). Therefore, it is required to estimate them by an adaptive law. The adaptive law is given in Section 4 .

Remark 8. The proposed approach does not provide the value function but the transformation coefficient is determined such that the modified ACLF (8) satisfies the HJB equation. Since the modified ACLF (8) depends on the original ACLF, the value of the cost function (17) varies depending on the selection of the original ACLF.

\section{Application of Theorem 2 to ACLF Obtained by Backstepping}

In this section, the transformation coefficient, the modified ACLF, and a nonlinear adaptive control law are designed by applying Theorem 2 to the ACLF which is chosen in the following backstepping. Backstepping can be applied to the strict-feedback system (1) by the following procedure [16].

Step $i(i=1, \ldots, n)$. We introduce the variables of $z_{i}(t)$, $\alpha_{i}\left(\bar{x}_{i}, \widehat{\theta}\right)$, and $\alpha_{n}\left(\bar{x}_{n}, \widehat{\theta}\right)$ defined by (23) and (24):

$$
\begin{gathered}
z_{i}(t) \stackrel{\Delta}{=} x_{i}(t)-\alpha_{i-1}\left(\bar{x}_{i-1}, \widehat{\theta}\right), \\
u_{B_{s}}(t)=\alpha_{n}\left(\bar{x}_{n}, \widehat{\theta}\right),
\end{gathered}
$$


where $\bar{x}_{i}=\left(x_{1}, \ldots, x_{i}\right), \alpha_{i}\left(\bar{x}_{i}, \widehat{\theta}\right) \in \mathbb{R}^{i+p} \rightarrow \mathbb{R}(i=1, \ldots$, $n-1)$ are the virtual inputs and $\alpha_{0}\left(\bar{x}_{0}, \hat{\theta}\right)=0$ and $u_{B_{s}}(t)$ denotes the control law derived in the step $n$ of backstepping. Differentiating (23) with respect to $t$ and using (1), $z$ system is written as

$$
\begin{aligned}
\dot{z}_{i}(t)= & f_{i}\left(\bar{x}_{i}\right)+F_{i}^{T}\left(\bar{x}_{i}\right) \theta-\dot{\alpha}_{i-1}\left(\bar{x}_{i-1}, \widehat{\theta}\right) \\
& +g_{i}\left(\bar{x}_{i}\right)\left(z_{i+1}(t)+\alpha_{i}\left(\bar{x}_{i}, \hat{\theta}\right)\right),
\end{aligned}
$$

where $z_{n+1}(t)=0$ and $\dot{\alpha}_{i}\left(\bar{x}_{i}, \widehat{\theta}\right)$ is given by

$$
\dot{\alpha}_{i}\left(\bar{x}_{i}, \widehat{\theta}\right)=\sum_{j=1}^{i} \frac{\partial \alpha_{i}\left(\bar{x}_{i}, \widehat{\theta}\right)}{\partial x_{j}} \dot{x}_{j}(t)+\left(\frac{\partial \alpha_{i}\left(\bar{x}_{i}, \widehat{\theta}\right)}{\partial \widehat{\theta}}\right)^{T} \dot{\hat{\theta}}(t) .
$$

If the $z$ system (25) is asymptotically stable, then the $x$ system (1) is also asymptotically stable. Therefore, we solve the problem of stabilization of the $z$ system by Lyapunov approach considering the Lyapunov function candidate as follows (in this paper, $\mathscr{V}^{\star}(\cdot, \widehat{\theta})$ denotes the ACLF derived by backstepping. $u^{\star}(t), k^{\star}(x, \widehat{\theta})$ denote the control law and the transformation coefficient based on $\mathscr{V}^{\star}(\cdot, \widehat{\theta})$, respectively):

$$
\mathscr{V}_{i}^{\star}(z, \widehat{\theta})=\frac{1}{2} \sum_{j=1}^{i} z_{j}^{2}(t)+\frac{1}{2}(\theta-\widehat{\theta}(t))^{T} \Gamma^{-1}(\theta-\widehat{\theta}(t)) .
$$

The virtual input $\alpha_{i}\left(\bar{x}_{i}, \widehat{\theta}\right)$ is chosen as

$$
\begin{aligned}
\alpha_{i}\left(\bar{x}_{i}, \hat{\theta}\right)= & -\frac{1}{g_{i}\left(\bar{x}_{i}\right)} \\
& \times\left[g_{i-1}\left(\bar{x}_{i-1}\right) z_{i-1}(t)+c_{i} z_{i}(t)\right. \\
& +f_{i}\left(\bar{x}_{i}\right)+w_{i}^{T}\left(\bar{x}_{i}, \hat{\theta}\right) \hat{\theta}(t) \\
& -\sum_{j=1}^{i-1}\left\{\frac{\partial \alpha_{i-1}\left(\bar{x}_{i-1}, \widehat{\theta}\right)}{\partial x_{j}}\right. \\
& -\sum_{j=1}^{i-1} z_{j}(t)\left(\frac{\partial \alpha_{j-1}\left(\bar{x}_{j-1}, \widehat{\theta}\right)}{\partial \widehat{\theta}}\right)^{T} \Gamma w_{i}\left(\bar{x}_{j}, \widehat{\theta}\right) \\
& \left.-\left(\frac{\partial \alpha_{i-1}\left(\bar{x}_{i-1}, \widehat{\theta}\right)}{\partial \widehat{\theta}}\right)^{T} \Gamma \sum_{j=1}^{i} w_{j}\left(\bar{x}_{j}, \widehat{\theta}\right) z_{j}(t)\right],
\end{aligned}
$$

where $z_{0}(t)=0$ and $c_{i} \in \mathbb{R}^{+}$is a design parameter and

$$
w_{i}\left(\bar{x}_{i}, \widehat{\theta}\right)=F_{i}\left(\bar{x}_{i}\right)-\sum_{j=1}^{i-1} \frac{\partial \alpha_{i-1}\left(\bar{x}_{i-1}, \hat{\theta}\right)}{\partial x_{j}} F_{j}\left(\bar{x}_{j}\right) .
$$

The time derivative of $\mathscr{V}_{n}^{\star}(z, \widehat{\theta})$ along the solution of (25) in the Step $i$ is given by

$$
\begin{aligned}
\dot{\mathscr{V}}_{n}^{\star}(z, \widehat{\theta})= & -\sum_{i=1}^{n} c_{i} z_{i}^{2}(t) \\
& -\left\{\sum_{i=1}^{n} z_{i}(t)\left(\frac{\partial \alpha_{i-1}\left(\bar{x}_{i-1}, \widehat{\theta}\right)}{\partial \widehat{\theta}}\right)^{T}\right. \\
& \left.+(\theta-\widehat{\theta}(t))^{T} \Gamma^{-1}\right\} \\
& \times\left(\dot{\hat{\theta}}(t)-\Gamma \sum_{i=1}^{n} w_{i}\left(\bar{x}_{i}, \widehat{\theta}\right) z_{i}(t)\right) .
\end{aligned}
$$

If the tuning function $\tau\left(\bar{x}_{n}, \widehat{\theta}\right)$ is chosen as

$$
\dot{\hat{\theta}}(t)=\Gamma \sum_{i=1}^{n} w_{i}\left(\bar{x}_{i}, \widehat{\theta}\right) z_{i}(t)
$$

then we get

$$
\dot{\mathscr{V}}_{n}^{\star}(z, \widehat{\theta})=-\sum_{i=1}^{n} c_{i} z_{i}^{2}(t)<0
$$

Thus, $\mathscr{V}_{n}^{\star}(z, \widehat{\theta})$ is an adaptive Lyapunov function for the $z$ system, the $z$ system is asymptotically stable, and the estimation error $\theta-\widehat{\theta}$ is stable; then $x$ system is asymptotically stable. Since the adaptive Lyapunov function and the asymptotic stabilizing control law are given, $\mathscr{V}_{n}^{\star}(z, \widehat{\theta})$ is an ACLF.

Applying Theorem 2 to ACLF (27), we obtain the control law $u^{*}(t)$ as

$$
u^{\star}(t)=-\frac{k^{\star}\left(\bar{x}_{n}, \hat{\theta}\right)}{2 r(x)} \mathscr{V}_{n_{x_{n}}}^{\star}\left(\bar{x}_{n}, \widehat{\theta}\right) g_{n}\left(\bar{x}_{n}\right)
$$

where the transformation coefficient $k^{\star}\left(\bar{x}_{n}, \widehat{\theta}\right)$ is given by

$$
\begin{gathered}
k^{\star}\left(\bar{x}_{n}, \widehat{\theta}\right)=2 r\left(\bar{x}_{n}\right) \frac{\sigma_{1}^{\star}\left(\bar{x}_{n}, \widehat{\theta}\right)+\sigma_{2}^{\star}\left(\bar{x}_{n}, \widehat{\theta}\right)}{\left(\mathscr{V}_{n_{x_{n}}}^{\star}\left(\bar{x}_{n}, \widehat{\theta}\right) g_{n}\left(\bar{x}_{n}\right)\right)^{2}} \\
\left(\mathscr{V}_{n_{x_{n}}}^{\star}\left(\bar{x}_{n}, \widehat{\theta}\right) g_{n}\left(\bar{x}_{n}\right) \neq 0\right), \\
k^{\star}\left(\bar{x}_{n}, \widehat{\theta}\right)=-\frac{q\left(\bar{x}_{n}\right)}{\sigma_{1}^{\star}\left(\bar{x}_{n}, \widehat{\theta}\right)}\left(\mathscr{V}_{n_{x_{n}}}^{\star}\left(\bar{x}_{n}, \widehat{\theta}\right) g_{n}\left(\bar{x}_{n}\right)=0\right),
\end{gathered}
$$

$$
k^{\star}\left(\bar{x}_{n}, \widehat{\theta}\right)=0 \quad\left(\bar{x}_{n}=0\right)
$$


and $\sigma_{1}^{\star}\left(\bar{x}_{n}, \widehat{\theta}\right)$ and $\sigma_{2}^{\star}\left(\bar{x}_{n}, \widehat{\theta}\right)$ are given by

$$
\begin{aligned}
& \sigma_{1}^{\star}\left(\bar{x}_{n}, \widehat{\theta}\right) \\
& =\sum_{i=1}^{n-1} \mathscr{V}_{n_{x_{i}}}^{\star}\left(\bar{x}_{n}, \widehat{\theta}\right) \\
& \times\left(f_{i}\left(\bar{x}_{i}\right)+F_{i}^{T}\left(\bar{x}_{i}\right) \hat{\theta}+g_{i}\left(\bar{x}_{i}\right) x_{i+1}(t)\right) \\
& +\mathscr{V}_{n_{x_{n}}}^{\star}\left(\bar{x}_{n}, \widehat{\theta}\right)\left(f_{n}\left(\bar{x}_{n}\right)+F_{n}^{T}\left(\bar{x}_{n}\right) \widehat{\theta}\right) \\
& -\left(\sum_{i=1}^{n}\left(x_{i}(t)-\alpha_{i-1}\left(\bar{x}_{i-1}, \widehat{\theta}\right)\right) \frac{\partial \alpha_{i-1}\left(\bar{x}_{i-1}, \hat{\theta}\right)}{\partial \widehat{\theta}}\right)^{T} \\
& \times \Gamma \sum_{i=1}^{n} w_{i}\left(\bar{x}_{i}, \widehat{\theta}\right)\left(x_{i}(t)-\alpha_{i-1}\left(\bar{x}_{i-1}, \widehat{\theta}\right)\right), \\
& \sigma_{2}^{\star}\left(\bar{x}_{n}, \widehat{\theta}\right) \\
& =\sqrt{\left(\sigma_{1}^{\star}\left(\bar{x}_{n}, \widehat{\theta}\right)\right)^{2}+\frac{q\left(\bar{x}_{n}\right)}{r\left(\bar{x}_{n}\right)}\left(\mathscr{V}_{n_{x_{n}}}^{\star}\left(\bar{x}_{n}, \widehat{\theta}\right) g_{n}\left(\bar{x}_{n}\right)\right)^{2}}
\end{aligned}
$$

from the following which is rewritten by (23) and (27):

$$
\begin{aligned}
\mathscr{V}_{n}^{\star}\left(\bar{x}_{n}, \widehat{\theta}\right)= & \frac{1}{2} \sum_{i=1}^{n}\left(x_{i}(t)-\alpha_{i-1}\left(\bar{x}_{i-1}, \widehat{\theta}\right)\right)^{2} \\
& +\frac{1}{2}(\theta-\widehat{\theta}(t))^{T} \Gamma^{-1}(\theta-\widehat{\theta}(t)) .
\end{aligned}
$$

Now, we show the stability of the system (2). The time derivative of (36) along the solution of (2) with the control input (33) yields

$$
\begin{aligned}
& \dot{\mathscr{V}}_{n}^{\star}\left(\bar{x}_{n}, \widehat{\theta}\right)=-\sigma_{2}^{\star}\left(\bar{x}_{n}, \widehat{\theta}\right) \quad\left(\mathscr{V}_{n_{x_{n}}}^{\star}\left(\bar{x}_{n}, \widehat{\theta}\right) g_{n}\left(\bar{x}_{n}\right) \neq 0\right), \\
& \dot{\mathscr{V}}_{n}^{\star}\left(\bar{x}_{n}, \hat{\theta}\right)=\sigma_{1}^{\star}\left(\bar{x}_{n}, \hat{\theta}\right) \quad\left(\mathscr{V}_{n_{x_{n}}}^{\star}\left(\bar{x}_{n}, \widehat{\theta}\right) g_{n}\left(\bar{x}_{n}\right)=0\right) .
\end{aligned}
$$

Since $\mathscr{V}_{n_{x_{n}}}^{\star}\left(\bar{x}_{n}, \widehat{\theta}\right) g_{n}\left(\bar{x}_{n}\right)=z_{n}(t) g_{n}\left(\bar{x}_{n}\right)$ and $z_{n}(t)=0,(37 \mathrm{~b})$ becomes

$$
\sigma_{1}^{\star}(z, \widehat{\theta})=-\sum_{i=1}^{n-1} c_{i} z_{i}^{2}(t)<0 \quad\left(\mathscr{V}_{n_{x_{n}}}^{\star}\left(\bar{x}_{n}, \widehat{\theta}\right) g_{n}\left(\bar{x}_{n}\right)=0\right)
$$

from (32). Now, it is shown that (36) is an adaptive Lyapunov function and the system is, therefore, asymptotically stable to the origin.

Finally, we show that $\widetilde{\mathscr{V}}\left(\bar{x}_{n}, \widehat{\theta}\right)$ which is related to $\mathscr{V}\left(\bar{x}_{n}, \widehat{\theta}\right)$ by (8) satisfies HJB equation. Using $\widetilde{\mathscr{V}}\left(\bar{x}_{n}, \widehat{\theta}\right)$ instead of
$\overline{\mathscr{V}}\left(\bar{x}_{n}, \widehat{\theta}\right)$, we obtain HJB equation (39) for the system (1) and (3) with the cost function (4):

$$
\begin{aligned}
\mathscr{H}\left(\bar{x}_{n}, \widehat{\theta}\right)= & q\left(\bar{x}_{n}\right)+k^{\star}\left(\bar{x}_{n}, \widehat{\theta}\right) \sum_{i=1}^{n-1} \mathscr{V}_{x_{i}}^{\star}\left(\bar{x}_{n}, \widehat{\theta}\right) \\
& \times\left(f_{i}\left(\bar{x}_{i}\right)+F_{i}^{T}\left(\bar{x}_{i}\right) \theta+g_{i}\left(\bar{x}_{i}\right) x_{i+1}(t)\right) \\
& +k^{\star}\left(\bar{x}_{n}, \widehat{\theta}\right) \mathscr{V}_{x_{n}}^{\star}\left(\bar{x}_{n}, \widehat{\theta}\right)\left(f_{n}\left(\bar{x}_{n}\right)+F_{n}^{T}\left(\bar{x}_{n}\right) \theta\right) \\
& +k^{\star}\left(\bar{x}_{n}, \widehat{\theta}\right) \sum_{i=1}^{n} \mathscr{V}_{\widehat{\theta}}^{\star}\left(\bar{x}_{n}, \widehat{\theta}\right) \Gamma \tau\left(\bar{x}_{n}, \widehat{\theta}\right) \\
& -\frac{1}{4 r\left(\bar{x}_{n}\right)}\left(k^{\star}\left(\bar{x}_{n}, \widehat{\theta}\right) \mathscr{V}_{x_{n}}^{\star}\left(\bar{x}_{n}, \widehat{\theta}\right) g_{n}\left(\bar{x}_{n}\right)\right)^{2} .
\end{aligned}
$$

By substituting (34a)-(34c) into (39), it is shown that HJB equation is satisfied.

From the above, it is confirmed that Theorem 2 is satisfied.

\section{Numerical Example}

The effectiveness of the proposed control law is shown by a numerical example of a system with an unknown parameter. The numerical simulation is done for the second order system (40) and the cost function (41) [10]:

$$
\begin{aligned}
& \dot{x}_{1}(t)=x_{2}(t) \\
& \dot{x}_{2}(t)=-x_{1}(t)\left(\frac{\pi}{2}+\arctan \left(5 x_{1}(t)\right)\right) \\
& \quad-\frac{5 x_{1}^{2}(t)}{2\left(1+25 x_{1}^{2}(t)\right)}+(3.5+\theta) x_{2}(t)+3 u(t), \\
& \mathscr{J}=\int_{0}^{\infty}\left(x_{2}^{2}(t)+u^{2}(t)\right) d t
\end{aligned}
$$

where the true value of the unknown parameter is $\theta=0.5$.

The value function of the optimal control problem for the system (40) with the cost function (41) is given by the following equation if the true value of $\theta$ in (40) is 0.5 (the value function of the HJB equation is given in the existing result [10]):

$$
\overline{\mathscr{V}}\left(\bar{x}_{2}\right)=x_{1}^{2}(t)\left(\frac{\pi}{2}+\arctan \left(5 x_{1}(t)\right)\right)+x_{2}^{2}(t)
$$

and the optimal control law is $\bar{u}(t)=-3 x_{2}(t)$ from (6).

On the other hand, using the ACLF which is given by (43) from (27),

$$
\begin{gathered}
\mathscr{V}_{2}^{\star}\left(\bar{x}_{2}\right)=\frac{1}{2}\left\{x_{1}^{2}(t)+\left(c_{1} x_{1}(t)+x_{2}(t)\right)^{2}\right. \\
\left.+\frac{1}{\Gamma}(\theta-\hat{\theta}(t))^{2}\right\},
\end{gathered}
$$


the control law is derived as (44) by backstepping:

$$
\begin{aligned}
u_{B_{s}}(t)=\frac{1}{3}\left\{x_{1}(t)\left(\frac{\pi}{2}+\arctan \left(5 x_{1}(t)\right)-c_{1} c_{2}-1\right)\right. \\
+\frac{5 x_{1}^{2}(t)}{2\left(1+25 x_{1}^{2}(t)\right)} \\
\left.-x_{2}(t)\left(c_{1}+c_{2}+3.5+\hat{\theta}(t)\right)\right\} .
\end{aligned}
$$

Then, applying Theorem 2 to ACLF (43), the proposed control is

$$
u^{\star}(t)=-\frac{3}{2} k^{\star}\left(\bar{x}_{2}\right)\left(c_{1} x_{1}(t)+x_{2}(t)\right)
$$

and the transformation coefficient is

$$
\begin{aligned}
& k^{\star}\left(\bar{x}_{2}\right) \\
& =2 \frac{\sigma_{1}^{\star}\left(\bar{x}_{2}, \widehat{\theta}\right)+\sqrt{\left(\sigma_{1}^{\star}\left(\bar{x}_{2}, \widehat{\theta}\right)\right)^{2}+\left\{3 x_{2}(t)\left(c_{1} x_{1}(t)+x_{2}(t)\right)\right\}^{2}}}{\left\{3\left(c_{1} x_{1}(t)+x_{2}(t)\right)\right\}^{2}} \\
& k^{\star}\left(\bar{x}_{2}\right)=-\frac{x_{2}^{2}}{\sigma_{1}^{\star}(x, \hat{\theta})} \quad\left(\mathscr{V}_{2_{x_{2}}}^{\star T}\left(\bar{x}_{2}\right) g\left(\bar{x}_{2}\right) g\left(\bar{x}_{2}\right)=0\right), \quad(46 \mathrm{a}) \\
& k^{\star}\left(\bar{x}_{2}\right)=0 \quad\left(\bar{x}_{2}=0\right), \\
& \sigma_{1}^{\star}\left(\bar{x}_{2}, \hat{\theta}\right) \quad \\
& =x_{1}(t) x_{2}(t)-\left(c_{1} x_{1}(t)+x_{2}(t)\right) \\
& \times\left\{x_{1}(t)\left(\frac{\pi}{2}+\arctan \left(5 x_{1}(t)\right)\right)+\frac{5 x_{1}^{2}(t)}{2\left(1+25 x_{1}^{2}(t)\right)}\right. \\
& \left.\quad-x_{2}(t)\left(c_{1}+3.5+\hat{\theta}(t)\right)\right\} .
\end{aligned}
$$

It should be noted that if $x_{1}(t) \neq 0$ and $x_{2}(t)=0$, then $q\left(\bar{x}_{2}\right)=0$. In this case, we need to show that $\sigma_{1}^{\star}\left(\bar{x}_{2}, \widehat{\theta}\right) \neq 0$ mentioned in Remark 6. Actually, we can obtain (48) from (47) when $x_{2}(t)=0$ :

$$
\begin{aligned}
\sigma_{1}^{\star}\left(\bar{x}_{2}, \hat{\theta}\right)= & -c_{1} x_{1}^{2}(t)\left(\frac{\pi}{2}+\arctan \left(5 x_{1}(t)\right)\right) \\
& -\frac{5 c_{1} x_{1}^{3}(t)}{2\left(1+25 x_{1}^{2}(t)\right)}<0 \quad(q(x)=0) .
\end{aligned}
$$

TABle 1: Cost $\mathscr{J}$.

\begin{tabular}{lcc}
\hline Optimal & Proposed & Backstepping \\
\hline 25.3 & 25.4 & 31.0 \\
\hline
\end{tabular}

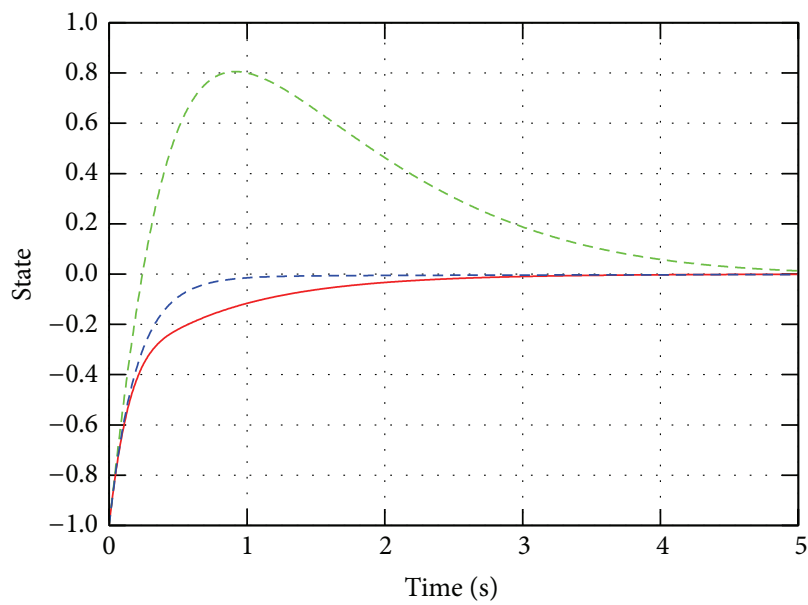

$$
\begin{aligned}
& - \text { Proposed } \\
& \text { FIgURE 1: Time histories of } x_{1}(t) .
\end{aligned}
$$

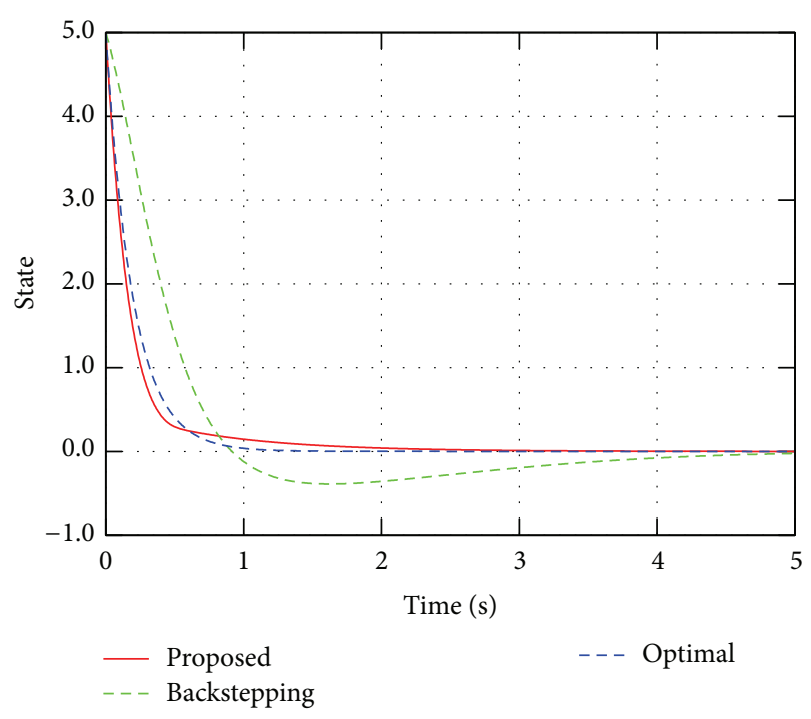

FIGURE 2: Time histories of $x_{2}(t)$.

Therefore, the proposed approach can be applied to ACLF (43).

The simulation results are shown in Figures 1, 2, 3, and 4. The initial state is $\bar{x}_{2}(0)=\left(\begin{array}{ll}-1.0 & 5.0\end{array}\right)$ and design parameters are $c_{i}=1$ and $\Gamma=1$. The value of the cost function $\mathscr{J}$ is shown in Table 1.

Figures 1-3 show that the trajectories by the proposed control law are close to the optimal trajectories and Table 1 shows that the value of the cost function by the proposed control law is smaller than the one by backstepping and close 


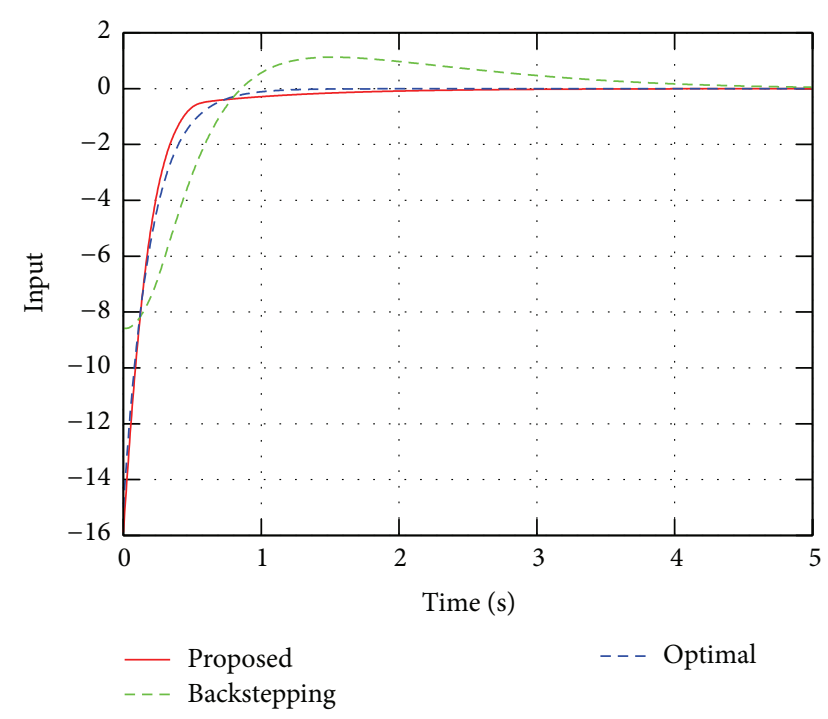

Figure 3: Time histories of $u(t)$.

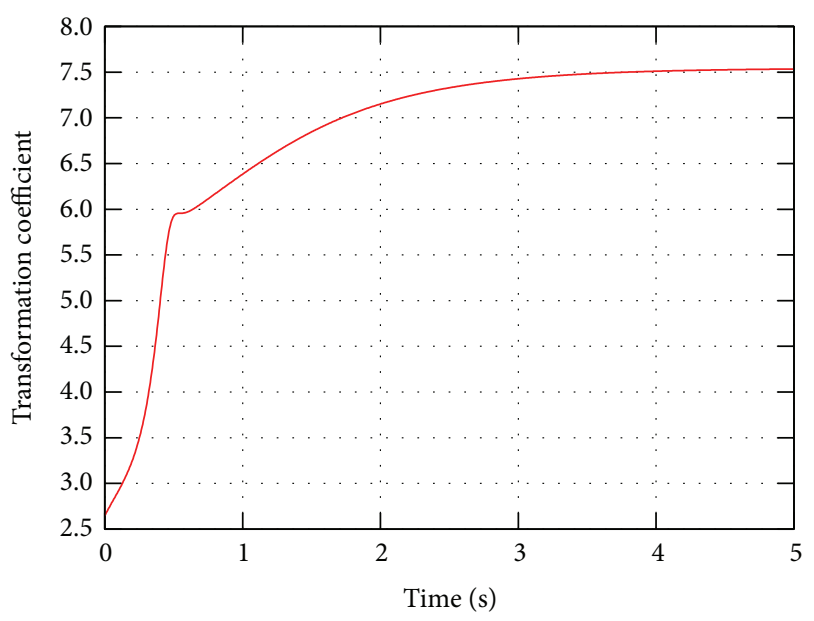

FIgURE 4: Time history of $k(x, \widehat{\theta})$.

to the optimal value for the system of which parameter $\theta$ is known.

\section{Conclusions}

In this paper, a nonlinear adaptive control law is proposed. The control law is designed by a modified ACLF which satisfies the HJB equation. It is proved that a cost function specified by a designer is minimized in the set of the control laws based on an ACLF for the strict-feedback system. The condition under which the proposed control law and the transformation coefficient are continuous is provided. The effectiveness of the proposed control law is shown by the simple numerical simulation.

If an ACLF is given, then the proposed approach enables designing a control law. Therefore, the proposed approach can also be applied to other nonlinear systems compared to strict-feedback systems.
Research subject in the future is extension to systems in which all the states cannot be accessed.

\section{Conflict of Interests}

The authors declare that there is no conflict of interests regarding the publication of this paper.

\section{References}

[1] A. E. Bryson and Y.-C. Ho, Applied Optimal Control-Optimization, Estimation and Control, Hemisphere Publishing, 1975.

[2] P. J. Moylan and B. D. Anderson, "Nonlinear regulator theory and an inverse optimal control problem," IEEE Transactions on Automatic Control, vol. 18, no. 5, pp. 460-465, 1973.

[3] S. T. Glad, "Robustness of nonlinear state feedback-A survey," Automatica, vol. 23, no. 4, pp. 425-435, 1987.

[4] R. A. Freeman and P. V. Kokotovic, "Inverse optimality in robust stabilization," SIAM Journal on Control and Optimization, vol. 34, no. 4, pp. 1365-1391, 1996.

[5] S. Kaizu and K. Hagino, "Nonlinear adaptive inverse optimal controller design considered optimality for nominal systems," The Transactions of The Institute of System, Control and Information Engineers, vol. 19, no. 4, pp. 123-131, 2006.

[6] H. Nakamura, Y. Sato, N. Nakamura, H. Katayama, and H. Nishitani, "Universal control formula for feedback linearizable systems with local LQ performance," in Proceedings of the European Control Conference, Budapest, Hungary, 2009.

[7] R. A. Freeman and J. A. Primbs, "Control Lyapunov functions: new ideas from an old source," in Proceedings of the 35th IEEE Conference on Decision and Control, pp. 3926-3931, Kobe, Japan, December 1996.

[8] J. L. Fausz, V.-S. Chellaboina, and W. M. Haddad, "Inverse optimal adaptive control for nonlinear uncertain systems with exogenous disturbances," in Proceedings of the 36th IEEE Conference on Decision and Control, pp. 2654-2659, San Diego, Calif, USA, December 1997.

[9] W. Luo, Y.-C. Chu, and K.-V. Ling, "Inverse optimal adaptive control for attitude tracking of spacecraft," IEEE Transactions on Automatic Control, vol. 50, no. 11, pp. 1639-1654, 2005.

[10] J. A. Primbs, V. Nevistic, and J. C. Doyle, "A receding horizon generalization of pointwise min-norm controllers," IEEE Transactions on Automatic Control, vol. 45, no. 5, pp. 898-909, 2000.

[11] H. Yuqing and H. Jianda, "Generalized point wise min-norm control based on control Lyapunov functions," in Proceedings of the 26th Chinese Control Conference (CCC '07), pp. 404-408, Hunan, China, July 2007.

[12] J. W. Curtis and R. W. Beard, "Satisficing: a new approach to constructive nonlinear control," IEEE Transactions on Automatic Control, vol. 49, no. 7, pp. 1090-1102, 2004.

[13] R. M. Milasi, M.-J. Yazdanpanah, and C. Lucas, "Nonlinear optimal control of washing machine based on approximate solution of HJB equation," Optimal Control Applications and Methods, vol. 29, no. 1, pp. 1-18, 2008.

[14] K. Okano and K. Hagino, "Adaptive control design approximating solution of Hamilton-Jacobi-Bellman equation for nonlinear strict-feedback system with uncertainties," in Proceedings of the SICE Annual Conference, pp. 204-208, IEEE, Tokyo, Japan, August 2008. 
[15] E. D. Sontag, Mathmatical Control Theory-Deterministic Finite Dimentional Systems, vol. 6 of Texts in Applied Mathematics, Springer, New York, NY, USA, 2nd edition, 1998.

[16] M. Krstic, I. Kanellakopoulos, and P. Kokotovic, Nonlinear and Adaptive Control Design, John Wiley \& Sons, New York, NY, USA, 1995. 

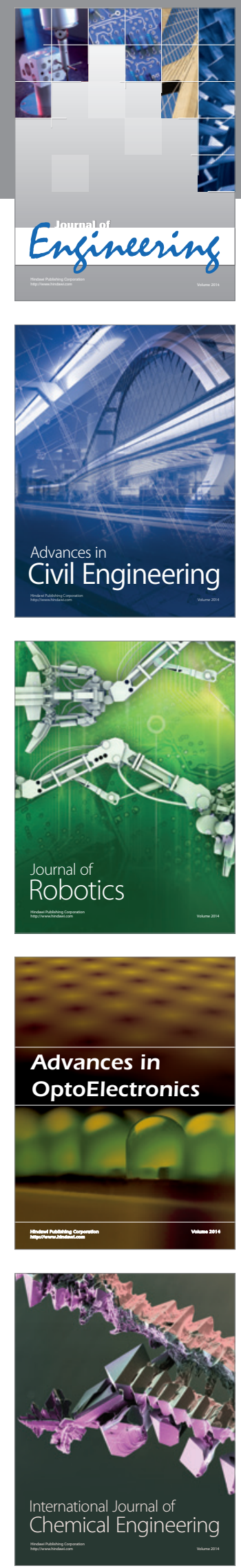

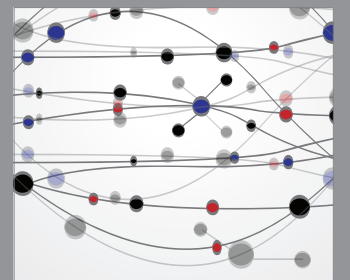

The Scientific World Journal
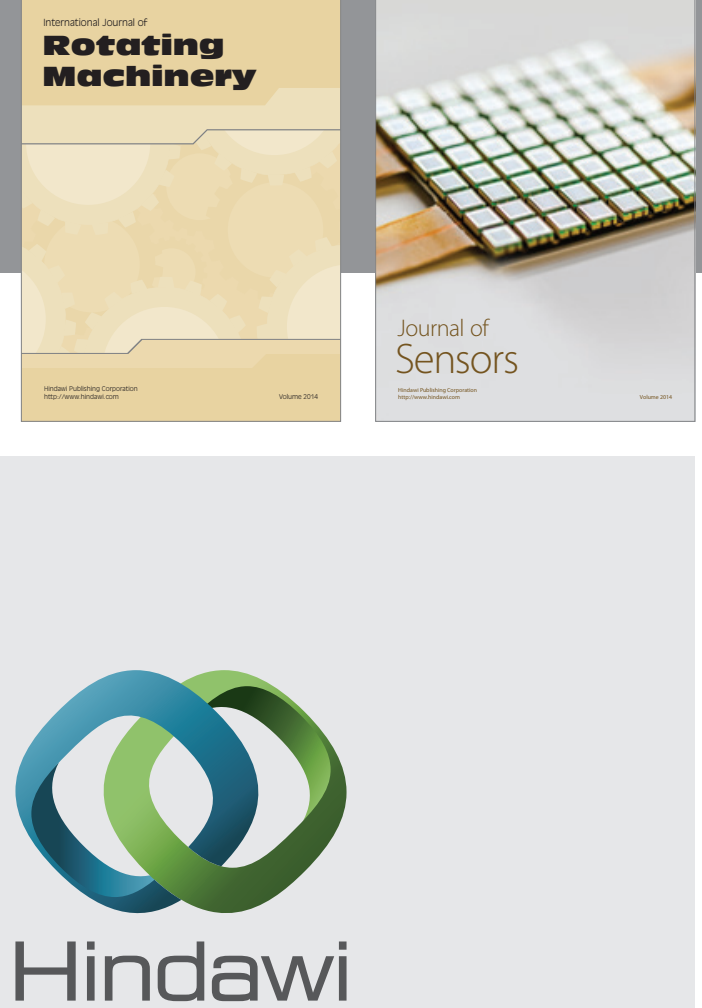

Submit your manuscripts at http://www.hindawi.com
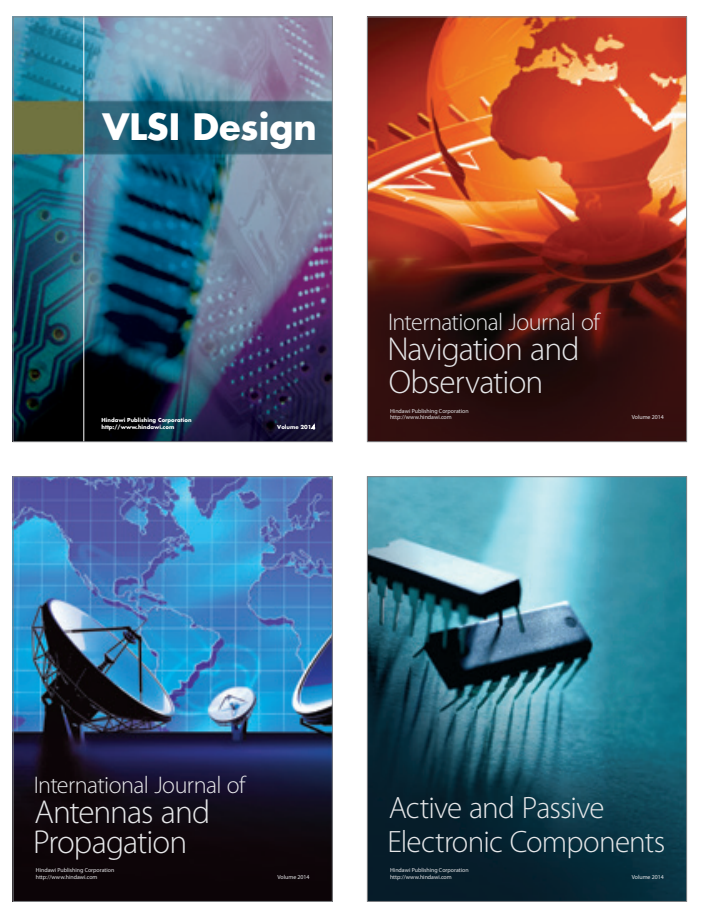
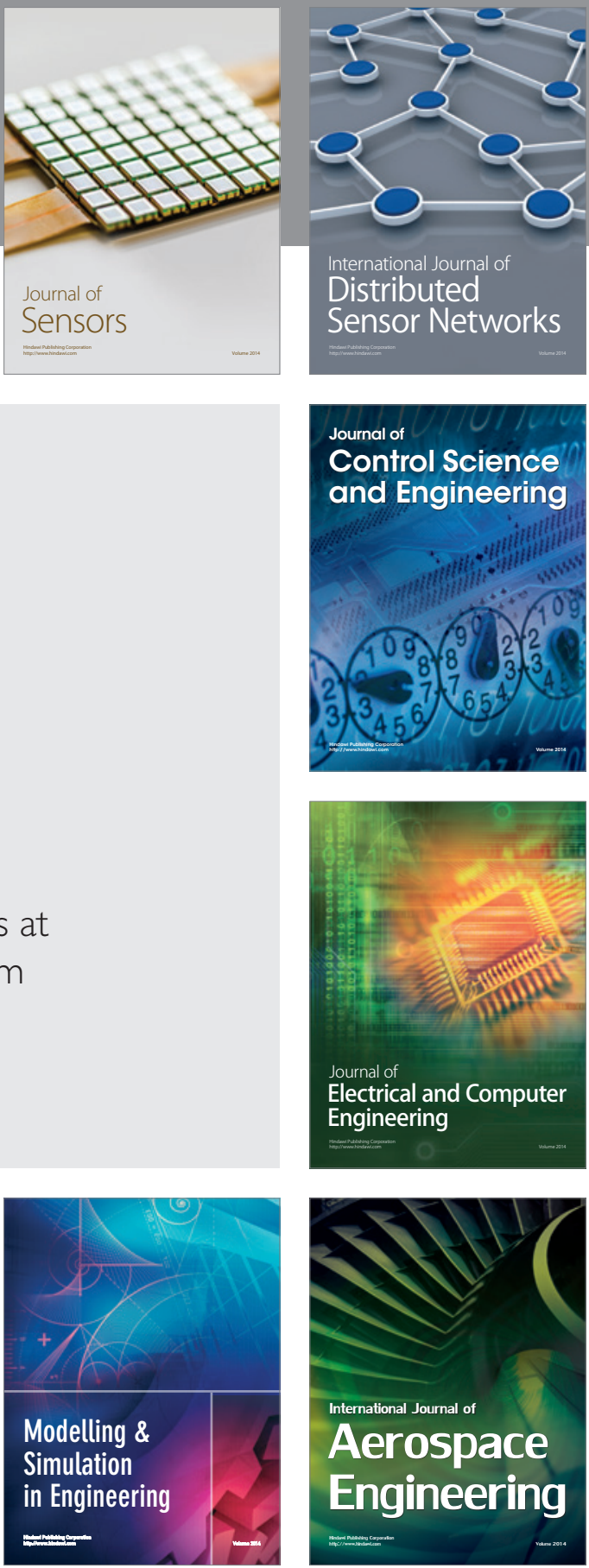

Journal of

Control Science

and Engineering
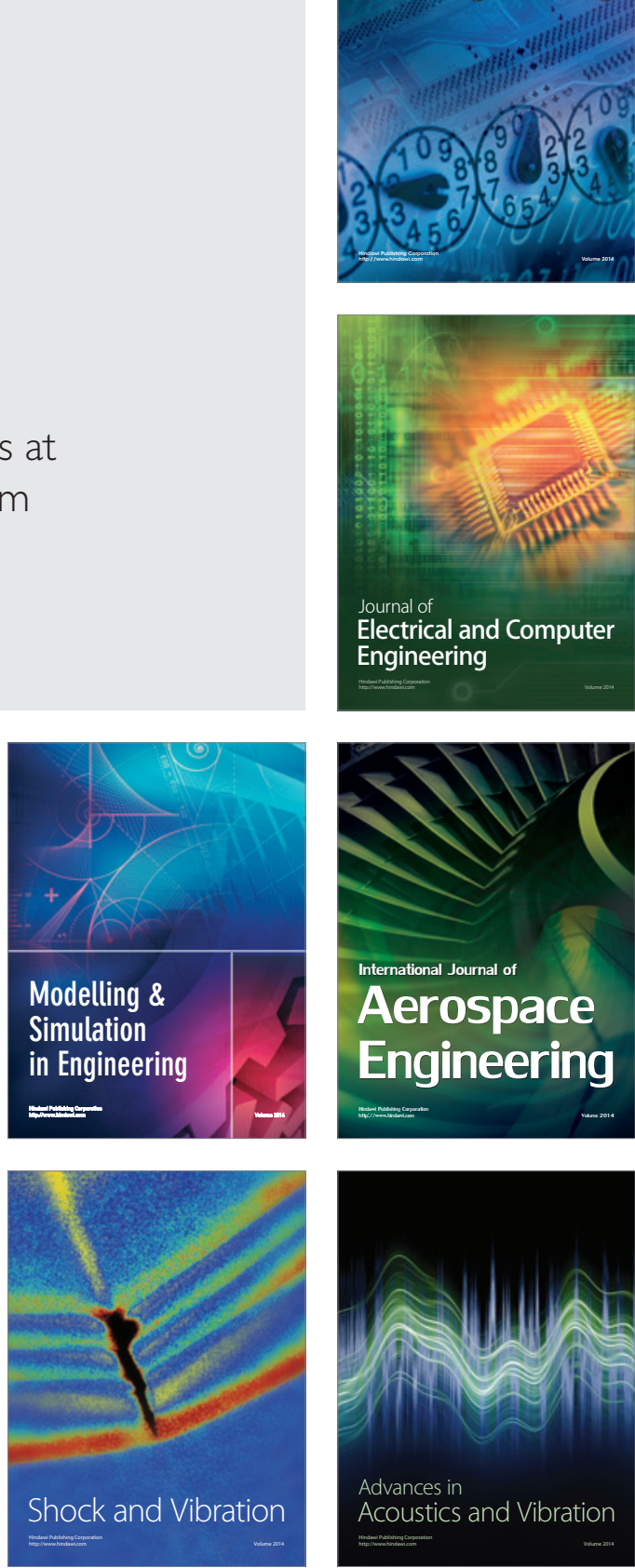